\title{
Causal Versus Clinical Interpretation
}

THE ARTICLE by Tierney and colleagues in this issue describes a strong association between hyponatremia and early death, and it may well make many readers uncomfortable. Some will be uncomfortable because no clear pathophysiologic mechanism for the role of hyponatremia in early death emerges. Some of the more epidemiologically sophisticated may be uncomfortable because they suspect that this may be due to some sort of selection or measurement bias, or, more likely, represent a secondary or confounded relationship. Still others will be uncomfortable trying to figure out what the authors mean when they conclude that "hyponatremia seems to be an indicator of underlying metabolic abnormalities that either directly cause death or contribute indirectly to increased mortality."

Epidemiologic reasoning involves two stages': The establishment of an unbiased association between $\alpha$ characteristic and a disease, which is unlikely to have occurred by chance, and the interpretation of the association in the context of other associations and knowledge. The latter stage is usually devoted to a "diagnosis" of causation. Clinical concerns and the perspective of clinical epidemiology suggest $\alpha$ third stage in this intellectual process, which is related to, but somewhat different from, the second. This stage has to do with assessing the potential utility of an association for improving clinical decision-making. ${ }^{2}$ The subtle differences in orientation and perspective between causal and clinical interpretation become apparent when considering the apparent risk of hyponatremia for death during hospitalization or shortly thereafter.

For purposes of discussion, let us assume that the increased mortality among individuals with admission serum sodiums of less than $130 \mathrm{mEq} / \mathrm{l}$ is not the result of bias in the selection of subjects or in the measurement of serum sodiums or mortality. Associations exclusively due to bias in study subject selection or the measurement of key variables are false or "spurious" and require no further interpretation.

We next need to know whether hyponatremia appears to be predictive of death only because its association in the data with another predictor of death has created a secondary or confounded ${ }^{3}$ association. In this regard, Anderson and colleagues ${ }^{4}$ reported that hyponatremic individuals in their hospital were 59 times more likely to die than normonatremic individuals. In their study, no variables other than serum sodium were included in the analysis. Tierney et al. report a relative risk of hospital death of 7.3. One likely explanation for this eightfold difference in the relative risks of hyponatremia in the two studies is that the former did not take into account any age differences between hyponatremic and normonatremic individuals, whereas the latter matched within two years of age.

Although additional analyses controlling for crude diagnostic categories did not further reduce the risk of hospital death, most readers would have been more comfortable if the authors had further information to control for more specific indicators of disease type and clinical severity. Few clinicians will be satisfied that crude diagnostic categories such as "pulmonary disease" and "diabetes" adequately account for important differences in the severity of illness and the likelihood of death, as has been recently demonstrated. ${ }^{5}$ Thus, we are left wondering whether hyponatremia, in and of itself, contributes to early death, or is rather a nonspecific concomitant of severe disease, $\alpha$ biochemical death rattle so to speak. Until such data are available, we cannot be certain whether the relationship between hyponatremia and early death is direct or is confounded by a relationship between hyponatremia and severe illness.

If the association were direct and not accounted for by disease severity or related factors, then the next step in epidemiologic reasoning would be to ask whether the association between hyponatremia and early death can be considered causal according to commonly accepted criteria. Here, causal and clinical reasoning overlap, since the prevailing view of causality has therapeutic implications. As stated in a classic epidemiologic text, "epidemiology has a practical purpose in the discovery of relationships which offer possibilities of disease prevention. For this purpose, therefore, $a$ causal relationship may be defined as an association between two categories of events in which $\alpha$ change in the frequency or quality of one is observed to follow alteration in the other." 6

The criteria for "diagnosing" causality proposed by Bradford Hill ${ }^{7}$ have been widely accepted. Application of these criteria to the association between hyponatremia and early death does not generate $\alpha$ strong case for a causal relationship, since there is at present no evidence of reversibility, $\alpha$ dose-response relationship, or a biologically plausible explanation for the apparent lethality of mild depressions in the admission serum sodium. Clearly, stronger and more diverse evidence as suggested by these criteria would be required by most of us before we would attempt to increase serum sodium in a patient with the asymptomatic, 
relatively mild hyponatremia described by Tierney et al.

But the same criteria are far less relevant in considering hyponatremia as a prognostic factor that appears to signal individuals at much greater risk of early death. In this case, we are less concerned with reversibility and pathophysiologic clarity than with performance. Does it consistently and validly predict which individuals are more likely to die in the hospital? The criteria by which we evaluate such clinical predictors are clearly different from those used to assess causation because the clinical purpose is different. A group of such criteria has been recently described. ${ }^{8}$ These criteria give emphasis to clinical accuracy, applicability, and utility rather than biological plausibility. The search for clinical predictors is an inelegant, utilitarian business where success is measured only by performance and old predictors will be discarded regularly as more refined and more predictive indicators are uncovered. Admission systolic blood pressure has proven to be a relatively crude indicator of cardiac pump failure, supplanted by more direct measures of cardiac performance, but it served clinicians well as a predictor of death in patients with myocardial infarction until better measures came along.

Understanding the future course of their illness is serious and importont business for patients, their families and their doctors. Research that contributes to this understanding must be encouraged whether it creatively uses existing datasets or builds new ones. Clearly, it must meet the same methodologic standards required of any modern epidemiologic inquiry, but its ultimate volue must be measured by the appropriate yardstick. Tierney and colleagues have nicely confirmed the clinical importance of hyponatremia. If the association proves to be causal, lives may be saved by prompt correction of the metabolic abnormality. If not causal, hyponatremia may still provide potent and useful information about the future. -Edward $H$. Wagner, MD, MPH, Director, Center for Health Studies, Group Health Cooperative of Puget Sound, Seattle, Washington

Acknowledgement: I am grateful to Dr. Thomas D. Koepsell for his helpful suggestions.

\section{REFERENCES}

1. Lilienfeld AM: Foundations of Epidemiology. New York: Oxford University Press, 1976;13-4

2. Spitzer WO: Clinical epidemiology (editorial). J Chronic Dis 1986:39:4115

3. Miettinen OS, Cook EF: Confounding: essence and detection. Am J Epidemiol 1981;114:593-603

4. Anderson RJ, Chung $H$, Kluge $R$, et al: Hyponatremia: a prospective analysis of its epidemiology and the pathogenic role of vasopressin. Ann Intern Med 1985;102:164-8

5. Charison ME, Sax FL, Mackenzie CR, et al: Assessing illness severity: does clinical judgment work? J Chronic Dis 1986;39:439-52

6. MacMahon B, Pugh TF: Epidemiology: principles and methods. Boston: Little, Brown and Company, 1970:17-8

7. Hill AB: Statistical evidence and inference. In: Principles of medical statistics. New York: Oxford University Press, 1966

8. Wasson JH, Sox HC, Neff RK, et al: Clinical prediction rules: applicaitons and methodological standards. N Engl J Med 1985;313:793-9

\section{Academic General Medicine and Motherhood:}

\section{In Search of a Balance}

WITH INCREASING NUMBERS of young women physicians entering academic medicine, medical centers must develop arrangements that allow these women to balance their academic careers and motherhood. This is likely to be particularly important for divisions of general medicine, as the number of young women faculty members in these divisions is often higher than in other divisions of departments of medicine. At Oregon Health Sciences University $15 \%$ of the Department of Medicine full-time faculty are women; half of these women are members of the division of general medicine.

Combining motherhood with a career is particularly a problem for faculty in general medicine since we tend to provide staffing for many clinical services $^{1}$ and assume heavy teaching loads. Patient care responsibilities have time demands that are unpredictable. At the same time, national trends show that academic general medicine faculty are coming under increasing pressure to publish. ${ }^{2}$ What happens when you add the time demands of motherhood to this already full schedule?

As two faculty members combining academic medicine and raising families, we have found the challenge demanding. Throughout our careers we have felt a strong desire to be "as good" as our male colleagues and to "pull our fair share" of the responsibilities. However, meeting our own standards of excellence in teaching, administration, patient care and clinical research became much harder following the births of our children. Returning to work full time within six weeks after childbirth was physically and emotionally difficult. The fatigue resulting from interrupted sleep and from nursing was greater than we had anticipated, and even greater than what we had experienced throughout much of our internships. 